\title{
Common summands in partitions
}

\author{
by \\ J. H. Loxton* and Hang-Fai Yeung (Sydney, N.S.W.)
}

1. Introduction. Erdős and Turán have made a number of forays in statistical group theory, investigating in particular the arithmetical structure of the symmetric group $S_{n}$ of order $n$. (See [2] and [3].) They posed many problems, as usual, one of these being the following question of Turán: Is it true that for almost all pairs of conjugacy classes of permutations of $S_{n}$, the cycle representations of the permutations in these classes contain cycles of the same length? From the one-to-one correspondence between conjugacy classes of $S_{n}$ and ordinary partitions of $n$, this amounts to the question whether or not almost all pairs of ordinary partitions of $n$ contain common summands.

Let $p(n)$ denote the number of ordinary partitions of $n$ and let $\Pi$ be a generic partition of $n$. For a partition $\Pi$, the set of its summands (with multiplicity) will be denoted by $\bar{\Pi}$ and the cardinality of $\bar{\Pi}$ by $|\bar{\Pi}|$. Turán [7] obtained the following result:

THEOREM 1. Let $\varepsilon>0$ be an arbitrarily small real number and $k \geq 2$ be a fixed integer. Suppose $n \leq n_{1} \leq n_{2} \leq \ldots \leq n_{k} \leq n(1+o(1))$ with $n \rightarrow \infty$. For sufficiently large $n$, the inequality

$$
\left|\bar{\Pi}_{1} \cap \ldots \cap \bar{\Pi}_{k}\right| \geq\left(\frac{1}{k}-\varepsilon\right) \max \left(\left|\bar{\Pi}_{1}\right|, \ldots,\left|\bar{\Pi}_{k}\right|\right)
$$

holds for almost all $k$-tuples $\Pi_{1}, \Pi_{2}, \ldots, \Pi_{k}$ of ordinary partitions of $n_{1}$, $n_{2}, \ldots, n_{k}$ respectively (that is with the exception of $o\left(p\left(n_{1}\right) p\left(n_{2}\right) \ldots p\left(n_{k}\right)\right)$ such $k$-tuples at most).

Essentially, the above theorem asserts that, for fixed $k$ and for almost all of the $k$-tuples of partitions in question, a positive percentage of summands occurs in all the $k$ partitions (independently of $n$ ). It is easy to see that for almost all partitions (that is, with the exception of $o(p(n))$ at most), the summand 1 appears at least $[\sqrt{n} / \omega(n)]$ times, where $\omega(n)$ is any function

\footnotetext{
* Research supported in part by a grant from the ARC.
} 
which tends to infinity with $n$. It seems reasonable to conclude that the above phenomenon is due to the presence of a large number of repeated small parts. However, this is not correct. For restricted partitions, Turán obtained a completely analogous result. Here, $q(n)$ denotes the number of restricted partitions of $n$.

Theorem 2. Let $\varepsilon, k, n, n_{1}, \ldots, n_{k}$ be as in Theorem 1 . For $n \rightarrow \infty$, the inequality

$$
\left|\bar{Q}_{1} \cap \ldots \cap \bar{Q}_{k}\right| \geq\left(\frac{1}{k 2^{k} \log 2}-\varepsilon\right) \max \left(\left|\bar{Q}_{1}\right|, \ldots,\left|\bar{Q}_{k}\right|\right)
$$

holds for almost all $k$-tuples $Q_{1}, Q_{2}, \ldots, Q_{k}$ of restricted partitions of $n_{1}$, $n_{2}, \ldots, n_{k}$ respectively (that is, with the exception of $o\left(q\left(n_{1}\right) q\left(n_{2}\right) \ldots q\left(n_{k}\right)\right)$ such $k$-tuples at most).

In [7], Turán claimed that inequalities like (1) and (2) can be obtained for partitions with summands taken from a general sequence of natural numbers. However, no extension in this direction has appeared in the literature. In this paper, we extend Theorem 2 to restricted partitions with summands taken from a wider class of sequences which includes the set of $s$ th powers. We go on to determine the distribution of the number of common parts in $k$-tuples of ordinary partitions under slightly more stringent conditions on the $n_{i}$ 's, namely $n \leq n_{1} \leq \ldots \leq n_{k} \leq n\left(1+o\left((\log n)^{-1-\delta}\right)\right)$ for some $\delta>0$. This resolves another problem of Turán under these conditions and shows that the constant $1 / k$ appearing in (1) is optimal.

2. Generalisation of the result of Turán. Let $\Lambda=\left\{\lambda_{1}, \lambda_{2}, \ldots\right\}$ be a strictly increasing sequence of positive integers. A restricted partition of $n$ is a partition of $n$ into distinct parts. A restricted $\Lambda$-partition of $n$ is a restricted partition of $n$ whose summands are taken from $\Lambda$. We denote the total number of restricted $\Lambda$-partitions of $n$ by $Q(n ; \Lambda)$.

In order to give asymptotic results for $Q(n ; \Lambda)$ and related quantities, it is necessary to put some restrictions on the sequence $\Lambda$. We shall suppose that $\Lambda$ satisfies the following two conditions (compare [3] and [5]):

$$
D_{\Lambda}(x)=\sum_{\substack{\lambda \operatorname{in}_{\lambda} \Lambda \\ \lambda \leq x}} 1=\frac{A x^{\alpha}}{\log ^{\beta} x}\left(1+O\left(\frac{1}{\log x}\right)\right),
$$

where $0<\alpha \leq 1$ and $\beta$ is real, and

$$
J_{k}=\inf \left\{\frac{1}{\log k} \sum_{\nu=1}^{k}\left\|\lambda_{\nu} \theta\right\|^{2}\right\} \rightarrow \infty
$$

as $k \rightarrow \infty$, where the infimum is taken over those $\theta$ satisfying $\frac{1}{2} \lambda_{k}^{-1}<\theta \leq \frac{1}{2}$. 
Notice that (I) implies that $\lim _{k \rightarrow \infty} \log \lambda_{k} / \log k=1 / \alpha$. Thus all the results obtained in [5] are applicable to a sequence $\Lambda$ satisfying (I) and (II). In particular, from equation (1) of [5], by a routine calculation,

$$
Q(n ; \Lambda)=\exp \left\{(1+o(1)) c_{1} n^{\alpha /(\alpha+1)} \log ^{-\beta /(\alpha+1)} n\right\},
$$

with $c_{1}=\left\{A \Gamma(\alpha+1) \zeta(\alpha+1)\left(1-2^{-\alpha}\right)(\alpha+1)^{\alpha+\beta+1} \alpha^{-\alpha}\right\}^{1 /(\alpha+1)}$ and $A$ is the constant in $(\mathrm{I})$.

The main point of the first part of this paper is to establish the following theorems generalising Theorem 2 above.

THEOREM 3. Let $\Lambda$ be a sequence of positive integers satisfying conditions (I) and (II) above. Let $k, n, n_{1}, \ldots, n_{k}$ be as in Theorem 1. For sufficiently large $n$, almost all $k$-tuples $Q_{1}, \ldots, Q_{k}$ of restricted $\Lambda$-partitions of $n_{1}, \ldots, n_{k}$ have at least

$$
\frac{1-o(1)}{2^{k} k^{\alpha}} c_{2} n^{\alpha /(\alpha+1)} \log ^{-\beta /(\alpha+1)} n
$$

common summands. Here $c_{2}=\left(\frac{A(\alpha+1)^{\beta} \Gamma(\alpha+1)}{\alpha^{\alpha} \zeta^{\alpha}(\alpha+1)\left(1-2^{-\alpha}\right)^{\alpha}}\right)^{1 /(\alpha+1)}$.

If, in addition, the partition function satisfies the inequality

(III) $\quad \log Q(n ; \Lambda)$

$$
>c_{1} n^{\alpha /(\alpha+1)} \log ^{-\beta /(\alpha+1)} n\left(1-\frac{1}{\log ^{1 /(2 \alpha+2)} n \log \log n}\right),
$$

then Erdős and Turán [3] have shown that almost all restricted $\Lambda$-partitions of $n$ contain

$$
c_{3} n^{\alpha /(\alpha+1)} \log ^{-\beta /(\alpha+1)} n\left(1+O\left(\log ^{-1 /(4 \alpha+4)} n\right)\right)
$$

summands, where

$$
c_{3}=\frac{A^{1 /(\alpha+1)} \Gamma(\alpha+1)^{1 /(\alpha+1)}\left(1-2^{1-\alpha}\right) \zeta(\alpha)(\alpha+1)^{\beta /(\alpha+1)}}{\left(\alpha\left(1-2^{-\alpha}\right) \zeta(\alpha+1)\right)^{\alpha /(\alpha+1)}} .
$$

(Note that, when $\alpha=1$, the indeterminate $\left(1-2^{1-\alpha}\right) \zeta(\alpha)$ is equal to $\log 2$.) In view of this, the following is an immediate consequence of Theorem 3.

THEOREM 4. Let $\Lambda$ be a sequence of positive integers satisfying the conditions (I), (II) and (III). Then, for any $\varepsilon>0, k \geq 2$ and for $n \rightarrow \infty$, almost all $k$-tuples $Q_{1}, \ldots, Q_{k}$ of restricted $\Lambda$-partitions of $n_{1}, \ldots, n_{k}$ with $n \leq n_{1} \leq \ldots \leq n_{k} \leq n(1+o(1))$ have at least

$$
\left(\frac{1}{2^{k} k^{\alpha}\left(1-2^{1-\alpha}\right) \zeta(\alpha)}-\varepsilon\right) \max \left(\left|\bar{Q}_{1}\right|, \ldots,\left|\bar{Q}_{k}\right|\right)
$$

common summands. 
It is known that the set of sth powers satisfies the three conditions (I), (II) and (III) and so provides a concrete example for Theorem 4. (See [3], p. 55.)

It is possible to work out the analogue of Theorem 3 for unrestricted $\Lambda$ partitions. However, even in the case of unrestricted partitions into squares, no analogue of Theorem 4 is known. The following question was put to the authors by Erdős:

Problem 1. Let $p_{2}(n)$ denote the number of unrestricted partitions of $n$ into squares. Does there exist a function $f(c)$ such that the number of unrestricted partitions of $n$ into squares in which the number of summands is less than $\mathrm{cn}^{2 / 3} \log n$ is asymptotic to $f(c) p_{2}(n)$ ?

3. Variation on a problem of Turán concerning common summands. Asymptotically, almost all ordinary partitions of $n$ have $\frac{\sqrt{6}}{2 \pi} \sqrt{n} \log n$ summands. Consequently, by Theorem 1 , the typical $k$-tuple of ordinary partitions of $n$ has asymptotically at least $\frac{\sqrt{6}}{2 \pi k} \sqrt{n} \log n$ common summands. This leads to the following problem of Turán.

Problem 2 (Turán [9]). Let $k \geq 2$ be a fixed integer and let $\lambda$ be a fixed real number. Suppose $n \leq n_{1} \leq n_{2} \leq \ldots \leq n_{k} \leq n(1+o(1))$. Denote by $K\left(n_{1}, \ldots, n_{k} ; \lambda\right)$ the number of $k$-tuples of ordinary partitions $\Pi_{1}, \ldots, \Pi_{k}$ of $n_{1}, \ldots, n_{k}$ with the property

$$
\left|\bar{\Pi}_{1} \cap \ldots \cap \bar{\Pi}_{k}\right| \leq \frac{\sqrt{6}}{2 \pi k} \sqrt{n} \log n+\lambda \sqrt{n} .
$$

Does there exist a distribution function $\Phi(\lambda)$ such that

$$
\lim _{n \rightarrow \infty} \frac{K\left(n_{1}, \ldots, n_{k} ; \lambda\right)}{p\left(n_{1}\right) \ldots p\left(n_{k}\right)}=\Phi(\lambda) ?
$$

In this section, we give an affirmative answer to a slight variation on this question. Our theorem settles the original problem when $\lambda=o(\log \log n)$ and $n \leq n_{1} \leq n_{2} \leq \ldots \leq n_{k} \leq n(1+\theta(n))$, where $\theta(n)=o\left((\log n)^{-1-\delta}\right)$ for some $\delta>0$.

Theorem 5. Let $k \geq 1$ be a fixed integer and $K\left(n_{1}, \ldots, n_{k} ; \lambda\right)$ be defined as above. Suppose $n \leq n_{1} \leq n_{2} \leq \ldots \leq n_{k} \leq n(1+\theta(n))$, where $\theta(n)=$ $o\left((\log n)^{-1-\delta}\right)$ for some $\delta>0$. Then, as $n \rightarrow \infty$,

$$
\frac{K\left(n_{1}, \ldots, n_{k} ; \lambda\right)}{p\left(n_{1}\right) \ldots p\left(n_{k}\right)} \sim \Phi(\lambda)=\exp \left(-\frac{1}{k d} e^{-k d \lambda}\right),
$$

where $d=\pi / \sqrt{6}$ and $\lambda=o(\log \log n)$.

Note that the case $k=1$ is the classical result of Erdös and Lehner [1]. Notice further that $\Phi(\lambda) \rightarrow 1$ as $\lambda \rightarrow \infty$ and $\Phi(\lambda) \rightarrow 0$ as $\lambda \rightarrow-\infty$. 
It follows from Theorem 5 that the number of common parts in almost all $k$-tuples of partitions of $n_{1}, \ldots, n_{k}$ (that is, with the exception of at most $o\left(p\left(n_{1}\right) \ldots p\left(n_{k}\right)\right)$ partitions) lies between the extremes $\frac{\sqrt{6}}{2 \pi k} \sqrt{n} \log n \pm$ $\omega(n) \sqrt{n}$, where $\omega(n) \rightarrow \infty$ with $n$ arbitrarily slowly. This, together with equation (1.4) in [1], shows that Theorem 1 is best possible in the sense that we cannot replace $1 / k$ in the theorem by a larger constant.

We are unable to obtain the analogue of the above theorem for restricted partitions and we propose the following problem:

Problem 3. Is the lower bound in (2) optimal? In particular, let $N\left(n_{1}\right.$, $\left.n_{2} ; \lambda\right)$ be the number of pairs of unequal partitions $Q_{1}, Q_{2}$ of $n_{1}, n_{2}$ such that $n \leq n_{1} \leq n_{2} \leq n(1+o(1))$ and $\left|\bar{Q}_{1} \cap \bar{Q}_{2}\right| \leq \frac{\sqrt{3}}{4 \pi} \sqrt{n}+\lambda n^{1 / 4}$. Is there $a$ distribution function $\Psi(\lambda)$ such that

$$
\lim _{n \rightarrow \infty} \frac{N\left(n_{1}, n_{2} ; \lambda\right)}{q\left(n_{1}\right) q\left(n_{2}\right)}=\Psi(\lambda) ?
$$

In view of Problem 1, it would be interesting to extend Theorem 5 to partitions with parts drawn from more general sequences as in Section 2. Our method fails because of the lack of a suitable generating function. (See equation (9) below.)

4. Proof of Theorem 3. In the course of the proof, we will need two simple lemmas which are consequences of hypothesis (I).

Lemma 1. Let $\Lambda$ be a sequence of positive integers satisfying (I). For $r \rightarrow 0^{+}$,

$$
\sum_{\lambda \text { in } \Lambda} e^{-r \lambda} \sim A \Gamma(\alpha+1) r^{-\alpha} \log ^{-\beta}\left(\frac{1}{r}\right) .
$$

Proof. For $r \rightarrow 0^{+}$, we can estimate

$$
\begin{aligned}
\sum_{\lambda \text { in } \Lambda} e^{-r \lambda} & =\int_{0}^{\infty} e^{-r x} d D_{\Lambda}(x)=\left[e^{-r x} D_{\Lambda}(x)\right]_{0}^{\infty}+r \int_{0}^{\infty} e^{-r x} D_{\Lambda}(x) d x \\
& =r \int_{2}^{\infty} \frac{A x^{\alpha} e^{-r x}}{\log ^{\beta} x}\left(1+O\left(\frac{1}{\log x}\right)\right) d x+O(r) .
\end{aligned}
$$

After substituting $y=r x$ in the integral, we see that the above is asymptotic to

$$
A r^{-\alpha} \log ^{-\beta}\left(\frac{1}{r}\right) \int_{0}^{\infty} e^{-y} y^{\alpha} d y
$$

and this is the required result. 
By a similar calculation, we also obtain the second lemma.

Lemma 2. $\operatorname{Let}_{\Lambda}(r)=\prod_{\lambda \text { in } \Lambda}\left(1+e^{-r \lambda}\right)$, with $\Lambda$ as in Lemma 1. For $r \rightarrow 0^{+}$,

$$
\log g_{\Lambda}(r) \sim c_{4} r^{-\alpha} \log ^{-\beta}\left(\frac{1}{r}\right),
$$

where $c_{4}=A\left(1-2^{-\alpha}\right) \Gamma(\alpha+1) \zeta(\alpha+1)$.

Now we proceed to the proof proper. Let $h_{\Lambda}\left(n_{1}, \ldots, n_{k}, n_{k+1}\right)$ denote the number of $k$-tuples of restricted $\Lambda$-partitions $Q_{1}, \ldots, Q_{k}$ of $n_{1}, \ldots, n_{k}$ respectively such that $\left|\bar{Q}_{1} \cap \ldots \cap \bar{Q}_{k}\right|=n_{k+1}$. We are done if we can show that whenever

$$
n_{k+1}<\frac{1-\varepsilon}{2^{k} k^{\alpha}} c_{2} n^{\alpha /(\alpha+1)} \log ^{-\beta /(\alpha+1)} n,
$$

we have

$$
\sum_{n=0}^{n_{k+1}} h_{\Lambda}\left(n_{1}, \ldots, n_{k}, n\right)=o\left(Q\left(n_{1} ; \Lambda\right) \ldots Q\left(n_{k} ; \Lambda\right)\right) .
$$

The generating function for $h_{\Lambda}$ is

$$
\begin{aligned}
\sum_{n_{1}, \ldots, n_{k+1}=0}^{\infty} h_{\Lambda}\left(n_{1}, \ldots,\right. & \left.n_{k}, n_{k+1}\right) x_{1}^{n_{1}} \ldots x_{k}^{n_{k}} t^{n_{k+1}} \\
& =\prod_{\lambda}\left\{\left(1+x_{1}^{\lambda}\right) \ldots\left(1+x_{k}^{\lambda}\right)-(1-t) x_{1}^{\lambda} \ldots x_{k}^{\lambda}\right\} .
\end{aligned}
$$

On putting $x_{i}=e^{-r_{i}}$ for $1 \leq i \leq k$ and $t=e^{-r_{k+1}}$, with each $r_{i}>0$, the above expression becomes

$$
\begin{aligned}
\sum_{n_{1}, \ldots, n_{k+1}=0}^{\infty} & h_{\Lambda}\left(n_{1}, \ldots, n_{k}, n_{k+1}\right) e^{-\left(n_{1} r_{1}+\ldots+n_{k+1} r_{k+1}\right)} \\
= & g_{\Lambda}\left(r_{1}\right) \ldots g_{\Lambda}\left(r_{k}\right) \prod_{\lambda}\left(1-\frac{\left(1-e^{-r_{k+1}}\right) e^{-\lambda\left(r_{1}+\ldots+r_{k}\right)}}{\left(1+e^{-\lambda r_{1}}\right) \ldots\left(1+e^{-\lambda r_{k}}\right)}\right) .
\end{aligned}
$$

Let us denote the last infinite product in (6) by $T(\boldsymbol{r})$. For $r_{i} \rightarrow 0^{+}$, we have

(7) $\log T(\boldsymbol{r})<-\left(1-e^{-r_{k+1}}\right) \sum_{\lambda} \frac{e^{-\lambda\left(r_{1}+\ldots+r_{k}\right)}}{\left(1+e^{-\lambda r_{1}}\right) \ldots\left(1+e^{-\lambda r_{k}}\right)}$

$$
\begin{aligned}
& \leq-\frac{1-e^{-r_{k+1}}}{2^{k}} \sum_{\lambda} e^{-\lambda\left(r_{1}+\ldots+r_{k}\right)} \\
& <-\frac{(1+o(1)) r_{k+1}}{2^{k}} A \Gamma(\alpha+1)\left(\sum_{i=1}^{k} r_{i}\right)^{-\alpha} \log ^{-\beta}\left(\sum_{i=1}^{k} r_{i}\right)^{-1}
\end{aligned}
$$


using Lemma 1. In (6), the coefficients $h_{\Lambda}\left(n_{1}, \ldots, n_{k}, n_{k+1}\right)$ are non-negative and so the sum of any group of terms from the left-hand side is less than the product on the right. In particular,

$$
\begin{aligned}
\sum_{n=0}^{n_{k+1}} h_{\Lambda}\left(n_{1}, \ldots, n_{k}, n\right) e^{-\left(n_{1} r_{1}+\ldots+n_{k+1} r_{k+1}\right)} & \\
< & \exp \left\{c_{4}(1+o(1))\left(\sum r_{i}^{-\alpha} \log ^{-\beta}\left(\frac{1}{r_{i}}\right)\right)\right. \\
& \left.\quad-\frac{(1+o(1)) r_{k+1}}{2^{k}} A \Gamma(1+\alpha)\left(\sum r_{i}\right)^{-\alpha} \log ^{-\beta}\left(\frac{1}{\sum r_{i}}\right)\right\} .
\end{aligned}
$$

(All the sums on the right run from $i=1$ to $k$.) Choose

$$
r_{i}=\frac{\alpha}{\alpha+1} c_{1} n_{i}^{-1 /(\alpha+1)} \log ^{-\beta /(\alpha+1)} n_{i} \quad(1 \leq i \leq k) .
$$

Rearranging (8) then gives

$$
\begin{aligned}
\sum_{n=0}^{n_{k+1}} h_{\Lambda}\left(n_{1}, \ldots, n_{k}, n\right) & \\
< & \exp \left\{\sum c_{1}(1+o(1)) n_{i}^{\alpha /(\alpha+1)} \log ^{-\beta /(\alpha+1)} n_{i}\right. \\
& \left.+r_{k+1}\left(n_{k+1}-\frac{1+o(1)}{2^{k} k^{\alpha}} c_{2} n^{\alpha /(\alpha+1)} \log ^{-\beta /(\alpha+1)} n\right)\right\} .
\end{aligned}
$$

If

$$
n_{k+1}<\frac{1-\varepsilon}{2^{k} k^{\alpha}} c_{2} n^{\alpha /(\alpha+1)} \log ^{-\beta /(\alpha+1)} n,
$$

$r_{k+1}$ is sufficiently small and fixed and $n$ is sufficiently large, then (3) gives

$$
\sum_{n=0}^{n_{k+1}} h_{\Lambda}\left(n_{1}, \ldots, n_{k}, n_{k+1}, n\right)=o\left(Q\left(n_{1} ; \Lambda\right) \ldots Q\left(n_{k} ; \Lambda\right)\right) .
$$

This completes the proof of Theorem 3 .

5. Common summands in a pair of partitions. We now begin the proof of Theorem 5. For simplicity, we give the details for the case $k=2$ and indicate the changes required for the general case later. More precisely, we shall prove the following theorem.

TheOREM 6. Let $K\left(n_{1}, n_{2} ; \lambda\right)$ denote the number of pairs of ordinary partitions $\Pi_{1}, \Pi_{2}$ of $n_{1}, n_{2}$ with the property

$$
\left|\bar{\Pi}_{1} \cap \bar{\Pi}_{2}\right| \leq \frac{\sqrt{6}}{4 \pi} \sqrt{n} \log n+\lambda \sqrt{n},
$$

where $\lambda=o(\log \log n), n \leq n_{1} \leq n_{2} \leq n(1+\theta(n))$ and $\theta(n)=o\left((\log n)^{-1-\delta}\right)$ 
for some $\delta>0$. Then, for $n \rightarrow \infty$,

$$
\frac{K\left(n_{1}, n_{2} ; \lambda\right)}{p\left(n_{1}\right) p\left(n_{2}\right)} \sim \exp \left(-\frac{1}{2 d} e^{-2 d \lambda}\right), \quad d=\frac{\pi}{\sqrt{6}} .
$$

Let $f(x)=\prod_{\nu=1}^{\infty}\left(1-x^{\nu}\right)^{-1}$ and $L=\left[\frac{\sqrt{6}}{4 \pi} \sqrt{n} \log n+\lambda \sqrt{n}\right]$. As shown in [7], pp. $193-195, K\left(n_{1}, n_{2} ; \lambda\right)$ is the coefficient of $x^{n_{1}} y^{n_{2}}$ in

$$
G(x, y)=f(x) f(y) \prod_{\nu=L+1}^{\infty}\left(1-(x y)^{\nu}\right)=\frac{f(x) f(y)}{f(x y)} \prod_{\nu=1}^{L} \frac{1}{1-(x y)^{\nu}} .
$$

Therefore, by Cauchy's theorem,

$$
K\left(n_{1}, n_{2} ; \lambda\right)=-\frac{1}{4 \pi^{2}} \iint \frac{G(z, w)}{z^{n_{1}+1} w^{n_{2}+1}} d z d w,
$$

where the integral is taken over the product of two circles $z=e^{-\alpha+i \theta}, w=$ $e^{-\beta+i \phi}$ with $-\pi<\theta, \phi \leq \pi$. Here, $\alpha$ and $\beta$ are chosen to satisfy the saddlepoint conditions

$$
\begin{aligned}
& \sum_{\nu=1}^{\infty} \frac{\nu}{e^{\alpha \nu}-1}-\sum_{\nu=L+1}^{\infty} \frac{\nu}{e^{(\alpha+\beta) \nu}-1}=n_{1}, \\
& \sum_{\nu=1}^{\infty} \frac{\nu}{e^{\beta \nu}-1}-\sum_{\nu=L+1}^{\infty} \frac{\nu}{e^{(\alpha+\beta) \nu}-1}=n_{2} .
\end{aligned}
$$

We choose

$$
\alpha=\frac{\pi}{\sqrt{6 n_{1}}}, \quad \beta=\frac{\pi}{\sqrt{6 n_{2}}} .
$$

By the Euler-Maclaurin formula, the two saddle-point equations hold up to errors of $O\left(\sqrt{n_{1}} \log ^{2} n_{1}\right)$ and $O\left(\sqrt{n_{2}} \log ^{2} n_{2}\right)$ respectively.

Now (10) can be written in the form

$$
K\left(n_{1}, n_{2} ; \lambda\right)=\frac{e^{\alpha n_{1}+\beta n_{2}}}{4 \pi^{2}} \int_{-\pi}^{\pi} \int_{-\pi}^{\pi} G\left(e^{-\alpha+i \theta}, e^{-\beta+i \phi}\right) e^{-i n_{1} \theta-i n_{2} \phi} d \theta d \phi
$$

Set $\theta_{0}=n^{-5 / 7}$. We split the double integral into four pieces: the main term $I_{1}$ corresponds to the major arc $|\theta| \leq \theta_{0},|\phi| \leq \theta_{0}$ and the error terms $I_{2}, I_{3}$ and $I_{4}$ correspond to the respective minor arcs $\theta_{0}<|\theta| \leq \pi, \theta_{0}<|\phi| \leq \pi$ for $I_{2}, \theta_{0}<|\theta| \leq \pi,|\phi| \leq \theta_{0}$ for $I_{3}$ and $|\theta| \leq \theta_{0}, \theta_{0}<|\phi| \leq \pi$ for $I_{4}$. The integrand in these double integrals can be written as

$$
\begin{aligned}
H(\theta, \phi)= & \exp \left\{-\sum_{\nu=1}^{\infty} \log \left(1-e^{-\alpha \nu}\right)-\sum_{\nu=1}^{\infty} \log \left(1-e^{-\beta \nu}\right)\right. \\
& \left.+\sum_{\nu=L+1}^{\infty} \log \left(1-e^{-(\alpha+\beta) \nu}\right)\right\} \exp \left\{S_{1}+S_{2}-i n_{1} \theta-i n_{2} \phi\right\}
\end{aligned}
$$


where

$$
\begin{gathered}
S_{1}=-\sum_{\nu=1}^{\infty} \log \left(\frac{1-e^{-\alpha \nu+i \nu \theta}}{1-e^{-\alpha \nu}}\right)-\sum_{\nu=1}^{\infty} \log \left(\frac{1-e^{-\beta \nu+i \nu \phi}}{1-e^{-\beta \nu}}\right), \\
S_{2}=\sum_{\nu=L+1}^{\infty} \log \left(\frac{1-e^{-(\alpha+\beta) \nu+i \nu(\theta+\phi)}}{1-e^{-(\alpha+\beta) \nu}}\right) .
\end{gathered}
$$

6. The major arc. Suppose $(\theta, \phi)$ lies in the region of integration of $I_{1}$. By a Taylor expansion,

$$
\begin{aligned}
S_{1}=i \theta \sum_{\nu=1}^{\infty} \frac{\nu}{e^{\alpha \nu}-1}+i \phi \sum_{\nu=1}^{\infty} \frac{\nu}{e^{\beta \nu}-1}-\frac{\theta^{2}}{2} \sum_{\nu=1}^{\infty} \frac{\nu^{2} e^{\alpha \nu}}{\left(e^{\alpha \nu}-1\right)^{2}} \\
\quad-\frac{\phi^{2}}{2} \sum_{\nu=1}^{\infty} \frac{\nu^{2} e^{\beta \nu}}{\left(e^{\beta \nu}-1\right)^{2}}+O\left(\frac{|\theta|^{3}}{\alpha^{4}}+\frac{|\phi|^{3}}{\beta^{4}}\right), \\
S_{2}=-i(\theta+\phi) \sum_{\nu=L+1}^{\infty} \frac{\nu}{e^{(\alpha+\beta) \nu}-1} \\
+\frac{(\theta+\phi)^{2}}{2} \sum_{\nu=L+1}^{\infty} \frac{\nu^{2} e^{(\alpha+\beta) \nu}}{\left(e^{(\alpha+\beta) \nu}-1\right)^{2}}+O\left(\frac{|\theta+\phi|^{3}}{(\alpha+\beta)^{4}}\right) .
\end{aligned}
$$

The contributions of the two error terms are at most $o(1)$. The saddle-point equations (11) are satisfied with error at most $O\left(\sqrt{n} \log ^{2} n\right)$, so the above expansion gives

$$
\begin{aligned}
H(\theta, \phi)=\exp & \left\{-\sum_{\nu=1}^{\infty} \log \left(1-e^{-\alpha \nu}\right)-\sum_{\nu=1}^{\infty} \log \left(1-e^{-\beta \nu}\right)\right. \\
& +\sum_{\nu=L+1}^{\infty} \log \left(1-e^{-(\alpha+\beta) \nu}\right) \\
& -\frac{\theta^{2}}{2} \sum_{\nu=1}^{\infty} \frac{\nu^{2} e^{\alpha \nu}}{\left(e^{\alpha \nu}-1\right)^{2}}-\frac{\phi^{2}}{2} \sum_{\nu=1}^{\infty} \frac{\nu^{2} e^{\beta \nu}}{\left(e^{\beta \nu}-1\right)^{2}} \\
& \left.+\frac{(\theta+\phi)^{2}}{2} \sum_{\nu=L+1}^{\infty} \frac{\nu^{2} e^{(\alpha+\beta) \nu}}{\left(e^{(\alpha+\beta) \nu}-1\right)^{2}}+o(1)\right\}
\end{aligned}
$$

Now

$$
\begin{aligned}
\sum_{\nu=L+1}^{\infty} \frac{\nu^{2} e^{(\alpha+\beta) \nu}}{\left(e^{(\alpha+\beta) \nu}-1\right)^{2}} & \leq 2 \sum_{\nu=L+1}^{\infty} \frac{\nu^{2}}{e^{(\alpha+\beta) \nu}} \sim 2 \int_{L}^{\infty} t^{2} e^{-(\alpha+\beta) t} d t \\
& \sim 2 e^{-(\alpha+\beta) L}\left(\frac{L^{2}}{\alpha+\beta}+\frac{2 L}{(\alpha+\beta)^{2}}+\frac{2}{(\alpha+\beta)^{3}}\right) .
\end{aligned}
$$


Since both $\alpha$ and $\beta$ are equal to

we have

$$
\frac{\pi}{\sqrt{6 n}}\left(1+o\left((\log n)^{-1-\delta}\right)\right)
$$

$$
(\alpha+\beta) L=\frac{1}{2} \log n+\frac{2 \pi}{\sqrt{6}} \lambda+o\left((\log n)^{-\delta}\right) .
$$

Also

$$
\frac{L^{2}}{\alpha+\beta} \sim c n^{3 / 2} \log ^{2} n, \quad \frac{L}{(\alpha+\beta)^{2}} \sim c n^{3 / 2} \log n, \quad \frac{1}{(\alpha+\beta)^{3}} \sim c n^{3 / 2}
$$

and so

$$
(\theta+\phi)^{2} \sum_{\nu=L+1}^{\infty} \frac{\nu^{2} e^{(\alpha+\beta) \nu}}{\left(e^{(\alpha+\beta) \nu}-1\right)^{2}} \leq c n^{-10 / 7} \cdot n^{-1 / 2} e^{-2 \pi \lambda / \sqrt{6}} \cdot n^{3 / 2} \log ^{2} n=o(1) .
$$

Here, and later, $c$ denotes an absolute positive constant which may vary from instance to instance. Hence, within the region of integration of $I_{1}$,

$$
\begin{aligned}
H(\theta, \phi)=\exp \{ & -\sum_{\nu=1}^{\infty} \log \left(1-e^{-\alpha \nu}\right)-\sum_{\nu=1}^{\infty} \log \left(1-e^{-\beta \nu}\right) \\
& \left.+\sum_{\nu=L+1}^{\infty} \log \left(1-e^{-(\alpha+\beta) \nu}\right)-\frac{A_{2} \theta^{2}}{2}-\frac{B_{2} \phi^{2}}{2}+o(1)\right\},
\end{aligned}
$$

where

$$
A_{2}=\sum_{\nu=1}^{\infty} \frac{\nu^{2} e^{\alpha \nu}}{\left(e^{\alpha \nu}-1\right)^{2}} \sim \frac{\pi^{2}}{3 \alpha^{3}}, \quad B_{2}=\sum_{\nu=1}^{\infty} \frac{\nu^{2} e^{\beta \nu}}{\left(e^{\beta \nu}-1\right)^{2}} \sim \frac{\pi^{2}}{3 \beta^{3}} .
$$

From all this, $A_{2} \theta_{0}^{2} \sim c n^{1 / 14}$ and $B_{2} \theta_{0}^{2} \sim c n^{1 / 14}$, so we can replace the limits of integration $\pm \theta_{0}$ in $I_{1}$ by $\pm \infty$ without altering the asymptotic estimation of $I_{1}$. Observe that

$$
\int_{-\infty}^{\infty} \int_{-\infty}^{\infty} \exp \left(-\frac{A_{2} \theta^{2}}{2}-\frac{B_{2} \phi^{2}}{2}\right) d \theta d \phi \sim \frac{\pi^{2}}{\sqrt{6} n_{1}^{3 / 4} n_{2}^{3 / 4}}
$$

Thus, finally,

$$
\begin{array}{r}
I_{1} \sim \frac{\pi^{2}}{\sqrt{6} n_{1}^{3 / 4} n_{2}^{3 / 4}} \exp \left\{-\sum_{\nu=1}^{\infty} \log \left(1-e^{-\alpha \nu}\right)-\sum_{\nu=1}^{\infty} \log \left(1-e^{-\beta \nu}\right)\right. \\
\left.+\sum_{\nu=L+1}^{\infty} \log \left(1-e^{-(\alpha+\beta) \nu}\right)\right\} .
\end{array}
$$

7. The minor arcs. We now show that $I_{2}, I_{3}$ and $I_{4}$ are negligible compared to $I_{1}$. 
To deal first with $I_{2}$, it suffices to show that

$$
J(\theta, \phi)=\exp \left\{S_{1}+S_{2}-i n_{1} \theta-i n_{2} \phi\right\}=o\left(n_{1}^{-3 / 4} n_{2}^{-3 / 4}\right)=o\left(n^{-3 / 2}\right)
$$

when $\theta_{0} \leq|\theta|,|\phi| \leq \pi$. The real parts of the sums $S_{1}$ and $S_{2}$ are given by

$$
\begin{aligned}
\operatorname{Re} S_{1}=-\frac{1}{2} \sum_{\nu=1}^{\infty} \log \left(1+\frac{4 e^{\alpha \nu} \sin ^{2}(\nu \theta / 2)}{\left(e^{\alpha \nu}-1\right)^{2}}\right) & \\
& -\frac{1}{2} \sum_{\nu=1}^{\infty} \log \left(1+\frac{4 e^{\beta \nu} \sin ^{2}(\nu \phi / 2)}{\left(e^{\beta \nu}-1\right)^{2}}\right)
\end{aligned}
$$

and

$$
\operatorname{Re} S_{2}=\frac{1}{2} \sum_{\nu=L+1}^{\infty} \log \left(1+\frac{4 e^{(\alpha+\beta) \nu} \sin ^{2}(\nu(\theta+\phi) / 2)}{\left(e^{(\alpha+\beta) \nu}-1\right)^{2}}\right) .
$$

The last sum is positive and bounded by

$$
\frac{1}{2} \sum_{\nu=L+1}^{\infty} \frac{4 e^{(\alpha+\beta) \nu}}{\left(e^{(\alpha+\beta) \nu}-1\right)^{2}} \leq 8 \sum_{\nu=L+1}^{\infty} e^{-(\alpha+\beta) \nu} \leq c(\alpha+\beta)^{-1} e^{-(\alpha+\beta) L} .
$$

But $e^{-(\alpha+\beta) L} \sim n^{-1 / 2} e^{-c \lambda}$ and $\lambda=o(\log \log n)$ by hypothesis, so $\left|\operatorname{Re} S_{2}\right| \leq$ $c e^{-c \lambda}=o\left(n^{\varepsilon}\right)$, for every $\varepsilon>0$. Thus

$$
\begin{aligned}
|J(\theta, \phi)| \leq \exp \left\{-\frac{1}{2} \sum_{\nu \leq \sqrt{n}} \log \left(1+\frac{4 e^{\alpha \nu} \sin ^{2}(\nu \theta / 2)}{\left(e^{\alpha \nu}-1\right)^{2}}\right)\right. \\
\left.-\frac{1}{2} \sum_{\nu \leq \sqrt{n}} \log \left(1+\frac{4 e^{\beta \nu} \sin ^{2}(\nu \phi / 2)}{\left(e^{\beta \nu}-1\right)^{2}}\right)+o\left(n^{\varepsilon}\right)\right\} .
\end{aligned}
$$

Following the argument in [6] or [4], pp. 267-269,

$$
|J(\theta, \phi)| \leq \exp \left(-c n_{1}^{1 / 14}-c n_{2}^{1 / 14}+o\left(n^{\varepsilon}\right)\right)=o\left(n_{1}^{-3 / 4} n_{2}^{-3 / 4}\right)
$$

when $\theta_{0} \leq|\theta|,|\phi| \leq \pi$, as required. This gives $\left|I_{2}\right|=o\left(I_{1}\right)$.

For $I_{3}$ and $I_{4}$, we can combine the techniques used for $I_{1}$ and $I_{2}$. Let $S_{\alpha}$ and $S_{\beta}$ denote the two constituent sums in $S_{1}$, that is

$$
S_{1}=-S_{\alpha}-S_{\beta} \text {. }
$$

Over the region of integration for $I_{3}$, namely $\theta_{0}<|\theta| \leq \pi,|\phi| \leq \theta_{0}$, we have

$$
\left|\exp \left(-S_{\alpha}+S_{2}-i n_{1} \theta\right)\right| \leq \exp \left(-c n^{1 / 14}\right),
$$

as in the treatment of $I_{2}$, and

$$
\int_{-\theta_{0}}^{\theta_{0}}\left|\exp \left(-S_{\beta}-i n_{2} \phi\right)\right| d \phi \sim \frac{\pi}{6^{1 / 4} n_{2}^{3 / 4}},
$$

as in the treatment of $I_{1}$. So $I_{3}=o\left(I_{1}\right)$ and similarly for $I_{4}$. 
8. Return to the major arc. By (12), (14) and the estimates of the last section,

$$
\begin{aligned}
& K\left(n_{1}, n_{2} ; \lambda\right) \\
& \quad \sim \frac{e^{\alpha n_{1}+\beta n_{2}}}{4 \sqrt{6} n_{1}^{3 / 4} n_{2}^{3 / 4}} \frac{f\left(e^{-\alpha}\right) f\left(e^{-\beta}\right)}{f\left(e^{-\alpha-\beta}\right)} \exp \left\{-\sum_{\nu=1}^{L} \log \left(1-e^{-(\alpha+\beta) \nu}\right)\right\},
\end{aligned}
$$

where $f(x)=\prod_{\nu=1}^{\infty}\left(1-x^{\nu}\right)^{-1}$. To estimate $f$, we use the well-known estimate

$$
f\left(e^{-x}\right)=\exp \left(\frac{\pi^{2}}{6 x}+\frac{1}{2} \log \frac{x}{2 \pi}+o(1)\right)
$$

as $x \rightarrow 0^{+}$. This gives

$$
\begin{aligned}
K\left(n_{1}, n_{2} ; \lambda\right) & \begin{aligned}
\sim \frac{e^{\alpha n_{1}+\beta n_{2}}}{4 \sqrt{6} n_{1}^{3 / 4} n_{2}^{3 / 4}} \exp & \left\{\frac{\pi^{2}}{6 \alpha}+\frac{1}{2} \log \frac{\alpha}{2 \pi}+\frac{\pi^{2}}{6 \beta}+\frac{1}{2} \log \frac{\beta}{2 \pi}-\frac{\pi^{2}}{6(\alpha+\beta)}\right. \\
& \left.-\frac{1}{2} \log \left(\frac{\alpha+\beta}{2 \pi}\right)-\sum_{\nu=1}^{L} \log \left(1-e^{-(\alpha+\beta) \nu}\right)\right\} .
\end{aligned}
\end{aligned}
$$

By the Euler-Maclaurin formula,

(18) $\sum_{\nu=1}^{L} \log \left(1-e^{-(\alpha+\beta) \nu}\right)=-\frac{\pi^{2}}{6(\alpha+\beta)}+\frac{e^{-(\alpha+\beta) L}}{\alpha+\beta}-\frac{1}{2} \log \frac{\alpha+\beta}{2 \pi}+o(1)$.

Also, from the choice of $\alpha$ and $\beta$ and the hypotheses on $n_{1}$ and $n_{2}$,

$$
\frac{1}{\alpha+\beta}=\left(\frac{\pi}{\sqrt{6 n_{1}}}+\frac{\pi}{\sqrt{6 n_{2}}}\right)^{-1}=\frac{\sqrt{6 n}}{2 \pi}\left(1+o\left((\log n)^{-1-\delta}\right)\right)
$$

and, by (13),

$$
\begin{aligned}
\frac{e^{-(\alpha+\beta) L}}{\alpha+\beta} & =\frac{\sqrt{6 n}}{2 \pi} \cdot \frac{e^{-2 \pi \lambda / \sqrt{6}}}{\sqrt{n}}\left(1+o\left((\log n)^{-\delta}\right)\right) \\
& =\frac{e^{-\pi \sqrt{2 / 3} \lambda}}{\pi \sqrt{2 / 3}}+o(1),
\end{aligned}
$$

since $\lambda=o(\log \log n)$. Substituting into (17) gives

$$
\begin{aligned}
K\left(n_{1}, n_{2} ; \lambda\right) & \sim \frac{1}{48 n_{1} n_{2}} \exp \left\{\frac{2 \pi}{\sqrt{6}}\left(\sqrt{n_{1}}+\sqrt{n_{2}}\right)-\frac{e^{-\pi \sqrt{2 / 3} \lambda}}{\pi \sqrt{2 / 3}}\right\} \\
& \sim p\left(n_{1}\right) p\left(n_{2}\right) \exp \left\{-\frac{e^{-\pi \sqrt{2 / 3} \lambda}}{\pi \sqrt{2 / 3}}\right\},
\end{aligned}
$$

which proves Theorem 6 . 
9. Common summands in $k$-tuples of partitions. To obtain Theorem 5 , we modify the argument of the preceding sections as follows. Set

$$
L_{k}=\left[\frac{\sqrt{6}}{2 \pi k} \sqrt{n} \log n+\lambda \sqrt{n}\right] .
$$

The generating function for $K\left(n_{1}, \ldots, n_{k} ; \lambda\right)$ is

$$
G_{k}\left(x_{1}, \ldots, x_{k}\right)=\frac{f\left(x_{1}\right) \ldots f\left(x_{k}\right)}{f\left(x_{1} \ldots x_{k}\right)} \prod_{\nu=1}^{L_{k}} \frac{1}{1-\left(x_{1} \ldots x_{k}\right)^{\nu}}
$$

and Cauchy's theorem yields

$$
K\left(n_{1}, \ldots, n_{k} ; \lambda\right)=\frac{1}{(2 \pi i)^{k}} \int \ldots \int \frac{G_{k}\left(z_{1}, \ldots, z_{k}\right)}{z_{1}^{n_{1}+1} \ldots z_{k}^{n_{k}+1}} d z_{1} \ldots d z_{k}
$$

where the integral is taken over the product of $k$ circles

$$
z_{i}=e^{-\alpha_{i}+i \theta_{i}}, \quad-\pi<\theta_{i} \leq \pi \quad(1 \leq i \leq k)
$$

and $\alpha_{1}, \ldots, \alpha_{k}$ are chosen to satisfy the saddle-point conditions

$$
\sum_{\nu=1}^{\infty} \frac{\nu}{e^{\alpha_{i} \nu}-1}-\sum_{\nu=L_{k}+1}^{\infty} \frac{\nu}{e^{\alpha \nu}-1}=n_{i} \quad(1 \leq i \leq k)
$$

and

$$
\alpha=\alpha_{1}+\ldots+\alpha_{k} .
$$

We choose $\alpha_{i}=\pi / \sqrt{6 n_{i}}$, so that the saddle-point conditions are satisfied up to an error $O\left(\sqrt{n} \log ^{2} n\right)$. This leads, as before, to

$$
\begin{aligned}
K\left(n_{1}, \ldots, n_{k} ; \lambda\right) \sim & \frac{1}{(2 \pi)^{k}} \int_{-\infty}^{\infty} \ldots \int_{-\infty}^{\infty} \exp \left(-\frac{1}{2} \sum_{i=1}^{k} A_{i} \theta_{i}^{2}\right) d \theta_{1} \ldots d \theta_{k} \\
& \times \exp \left\{\sum_{i=1}^{k} \alpha_{i} n_{i}-\sum_{i=1}^{k} \sum_{\nu=1}^{\infty} \log \left(1-e^{-\alpha_{i} \nu}\right)\right. \\
& \left.+\sum_{\nu=1}^{\infty} \log \left(1-e^{-\alpha \nu}\right)-\sum_{\nu=1}^{L_{k}} \log \left(1-e^{-\alpha \nu}\right)\right\},
\end{aligned}
$$

where

$$
A_{i}=\sum_{\nu=1}^{\infty} \frac{\nu^{2} e^{\alpha_{i} \nu}}{\left(e^{\alpha_{i} \nu}-1\right)^{2}} \sim \frac{\pi^{2}}{3 \alpha_{i}^{3}} \quad(1 \leq i \leq k) .
$$

The various sums here can be estimated by (16) and (18), leading finally to

$$
K\left(n_{1}, \ldots, n_{k} ; \lambda\right) \sim p\left(n_{1}\right) \ldots p\left(n_{k}\right) \exp \left(-\frac{e^{-\pi k \lambda / \sqrt{6}}}{\pi k / \sqrt{6}}\right) .
$$




\section{References}

[1] P. Erdős and J. Lehner, The distribution of the number of summands in the partitions of a positive integer, Duke Math. J. 8 (1941), 335-345.

[2] P. Erdős and P. Turán, On some problems of a statistical group theory VII, Period. Math. Hungar. 2 (1972), 149-163.

[3] - - - On some general problems in the theory of partitions, I, Acta Arith. 18 (1971), 53-62.

[4] H. Gupta, Selected Topics in Number Theory, Abacus Press, 1980.

[5] K. F. Roth and G. Szekeres, Some asymptotic formulae in the theory of partitions, Quart. J. Math. (Oxford) (2) 5 (1954), 244-259.

[6] G. Szekeres, Some asymptotic formulae in the theory of partitions (II), ibid. 4 (1953), 96-111.

[7] P. Turán, Combinatorics, partitions, group theory, in: Colloquio Int. s. Teorie Combinatorie, Roma 1973, Accademia Nazionale dei Lincei, 1976, Tomo II, 181-200.

[8] —, On a property of partitions, J. Number Theory 6 (1974), 405-411.

[9] - On some phenomena in the theory of partitions, Astérisque 24-25 (1975), 311319 .

[10] - On some connections between combinatorics and group theory, in: Combinatorial Theory and its Applications (Balatonfüred 1969), Colloq. Math. Soc. J. Bolyai 4, North-Holland, 1970, 1055-1082.

SCHOOL OF MATHEMATICS, PHYSICS, COMPUTING AND ELECTRONICS

MACQUARIE UNIVERSITY

SYDNEY, NEW SOUTH WALES 2109

AUSTRALIA

Received on 5.3.1990

and in revised form on 24.1.1991 